\title{
Connectivity calculus of fractal polyhedrons
}

\author{
Helena Molina-Abril ${ }^{a, *}$, Pedro Real $^{\mathrm{a}}$, Akira Nakamura ${ }^{\mathrm{b}}$, Reinhard Klette ${ }^{\mathrm{c}}$ \\ a Universidad de Sevilla, Spain \\ ${ }^{\mathrm{b}}$ Hiroshima University, Japan \\ ${ }^{\mathrm{c}}$ The University of Auckland, New Zealand
}

Keywords:

Connectivity

Cycles

Topological analysis

Tunnels

Directed graphs

Betti number

Fractal set

Menger sponge

Sierpiński pyramid

\begin{abstract}
A B S T R A C T
The paper analyzes the connectivity information (more precisely, numbers of tunnels and their homological (co)cycle classification) of fractal polyhedra. Homology chain contractions and its combinatorial counterparts, called homological spanning forest (HSF), are presented here as an useful topological tool, which codifies such information and provides an hierarchical directed graph-based representation of the initial polyhedra. The Menger sponge and the Sierpiński pyramid are presented as examples of these computational algebraic topological techniques and results focussing on the number of tunnels for any level of recursion are given. Experiments, performed on synthetic and real image data, demonstrate the applicability of the obtained results. The techniques introduced here are tailored to selfsimilar discrete sets and exploit homology notions from a representational point of view. Nevertheless, the underlying concepts apply to general cell complexes and digital images and are suitable for progressing in the computation of advanced algebraic topological information of 3-dimensional objects.
\end{abstract}

\section{Introduction}

The Polish mathematician Waclaw Sierpiński described properties of a self-similar planar set (see [26]), today known as the Sierpinski sieve. A generalization into 3-dimensional space leads to the Sierpiński pyramid. The Austrian mathematician Karl Menger also studied a recursively defined set, today known as Menger sponge (see [16] and Fig. 1), when discussing the notion of topological dimension.

In this paper we consider recursively defined polyhedra, called fractal sets. Recursion details are given below. The Menger sponge or Sierpiński pyramid are examples of such fractal sets.

Working within a semi-continuous context, we use the notion of a minimal spanning tree (MST) of a finite set of points in $\mathbb{R}^{n}$, as introduced in $[22,23]$, to provide topological information of the fractal underlying set, up to a numerically computable resolution.

In particular, connectedness, disconnectedness and the number of connected components with non-zero diameter are properties that are identified and calculated in [23]. A logical question that arises here when investigating ways to formalize the relationship between the homology of a set of $\mathbb{R}^{3}$, and the homology of a finite point-set approximation of it, is how to distinguish between

\footnotetext{
* Corresponding author.

E-mail addresses: habril@us.es (H. Molina-Abril), real@us.es (P. Real), an1206@ad.cyberhome.ne.jp (A. Nakamura), r.klette@auckland.ac.nz (R. Klette).
}

simply-connected sets and those with holes (i.e. tunnels or cavities).

Within the semi-continuous context of cell complexes, there are two approaches for the computation of comprehensive homological information:

1. The (co)differential approach: Here, only one 2-nilpotent linear map (the canonical differential operator of the cell complex) is involved, and linear algebra for reducing matrices into a Smith Normal Form is exhaustively used (see e.g $[12,19]$ ).

2. The integral approach: Here, two 2-nilpotent linear maps (differential and integral operators) are involved. In the integral approach, one constructs a degree +1 linear map (integral operator) which records the information of the algebraic homological deformation process, reducing the whole cell complex to a minimal homological expression (see e.g. [10]).

In particular, using the differential and integral operators, one can determine homology groups among other topological properties.

For example, the method of computing connected components using the spanning forest of the 1-skeleton of the cell complex is a relevant example of integral homological computation. We apply here an integral homological approach, allowing a quantitative analysis of high degrees of connectivity of cell complex versions of fractals, for an arbitrary level of recursion. We mainly focus on the 


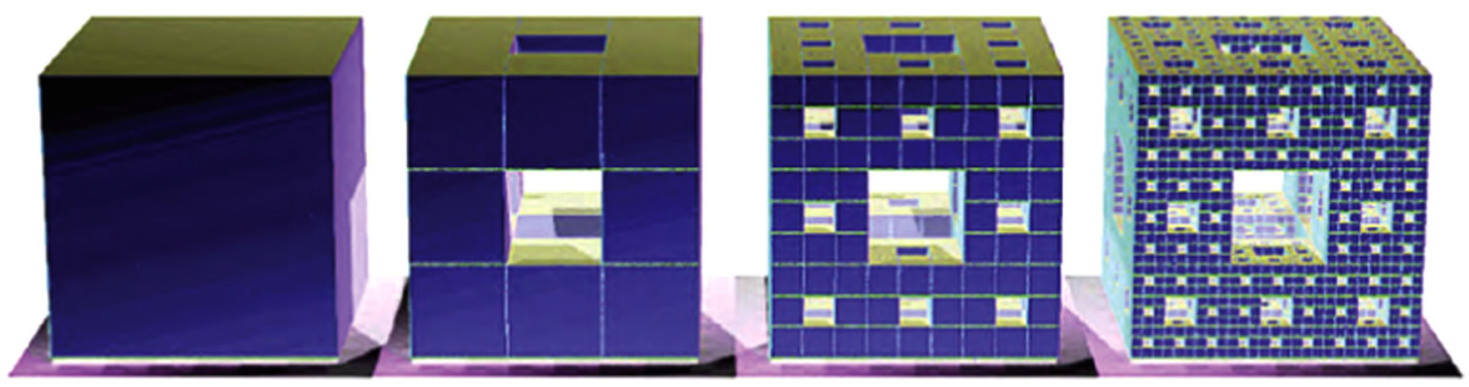

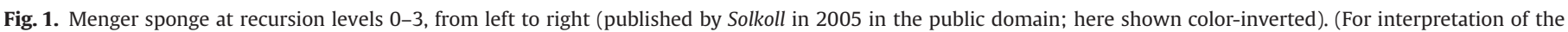
references to color in this figure caption, the reader is referred to the web version of this paper.)

number of tunnels and cavities, but the techniques developed here can be employed to create a calculus with cycles and co-cycles within the fractals.

A cycle calculus allows us, for example,

1. to topologically classify any cycle or co-cycle within the object,

2. to determine whether a cycle is contractible within the object and, in the positive case, to obtain a geometric deformation that reduces the cycle into a point,

3. to topologically transform, if possible, one cycle into another within the fractal,

4. to determine a shortest path between two points in the given fractal, considering a defined distance function, and

5. to compute (co-)cyclic operations having (co-)cycles of the objects as input or output.

Starting with a hierarchical directed graph representation, it is possible to proceed with cycle-calculus on fractals. This calculus has been introduced in $[17,18]$, by defining coordinate-based forests (i.e. graphs which are in general a set of trees). These direct graphs extent to higher dimensions spanning forests as known from labelling of connected components (0th-homology group) of a digital object. Due to this fact, they are called homological spanning forests (HSFs). These structures can also be generalized for applications in the domain of tree-cotree decompositions of combinatorial surfaces; see [6] and [7].

In this paper we recall the definition of HSFs, give relevant examples for fractal sets, and manipulate their associated chainhomotopy equivalence for computing topological properties. For developing this approach, different methodologies and theories have been combined, such as discrete Morse theory (see [8]), effective homology (see [25]), AT-model theory (see [10]), and homological algebra (see [17]).

The techniques introduced here are tailored to fractals, but the underlying concepts also apply to digital images and data. The development of new topological representations is essential for advancing in solid and physical modelling $[3,4]$. It appears to be a feasible short-term goal to design a novel mapping from mathematical solid models to actual computer representations for extensive geometric data as common in 3D imaging, using the HSF techniques as developed. See the reported experiments at the end of the paper.

Furthermore, digital topology has various applications in remote sensing, computer vision, lossless and fractal compression, and algorithmic pattern recognition. Because our method applies to fractal and non-fractal cell complexes, the work presented here is susceptible to be adapted for its use in the previously mentioned applications.

The paper is structured as follows: Section 2 introduces HSFs and related basic definitions. Section 3 applies the introduced topological framework to fractal polyhedra in general. The example of the Menger sponge is discussed in Section 4, and those of the Sierpiński pyramid in Section 5. Experiments are shown in Section 6. Section 7 concludes.

\section{Combining homological with homotopical information}

We determine the representative cycles of tunnels in fractal structures which are embedded into $\mathbb{R}^{3}$. By using HSF structures, we classify any closed curve in the fractal structure in terms of homology generators working with coefficients in the field $\mathbb{Z} / 2 \mathbb{Z}=\{0,1\}$.

A cell complex $O$ consists of $i$-cells of dimension $i$, for some $i \in \mathbb{N}$. Given a finite convex cell complex embedded in $\mathbb{R}^{3}$, it is possible to construct the canonical chain complex associated to a cell complex $O$ and its homology, called a strong deformation retract in [11], a reduction in [25], or a chain contraction in [5]. In fact, a chain contraction of this kind, called homology chain-integral contraction (See [20]), condenses the $\mathbb{Z} / 2 \mathbb{Z}$ homological information of the cell complex $O$ and it can be completely described by only using two chain operators. One of them is the (unique) chain boundary operator for $O$. The other is a (non-unique) twonilpotent chain homotopy called chain integral operator.

In order to take advantage of the geometric nature of the cell complex, we combine homological and homotopical information of $O$, for constructing the previous homology chain contraction by using the combinatorial boundary operator of $O$ and directed graphs whose nodes are the centroids of the (convex) cells. For example, if the homotopical information is given in terms of a gradient vector field $V$ on the cell complex $O$ (see $[8,14]$ ), it is straightforward to construct a chain contraction from the chain complex $O$ to a smaller (in terms of numbers of basis generators) algebraic object. In the case having an optimal vector field $V$, this last object is the $\mathbb{Z} / 2 \mathbb{Z}$-homology graded module of $O$. Summing up, we search here for an "economical" combinatorial coding of a homology chain integral operator (also called, AT-model, [9]). This "coding" will be given here in terms of hierarchical coordinate-based directed graphs, called homological spanning forest (HSF). This paper discusses the constructions of HSF structures for some fractal sets, to be used for determining homology chain contractions.

The directed edges of an HSF are part of the frontier of the cell complex which consists of several "connected" cell pairings. A cell pairing is a directed edge which goes from an $i$-cell to an incident $(i+1)$-cell of the complex. Let us note that in a general HSF, two cell pairings could share the $i$-cell or the $(i+1)$-cell components. The links between these cell pairings are also directed edges from the $(i+1)$-cell tail of one cell pairing to the $i$-cell source of the other. When the HSF is derived from a optimal discrete gradient vector field $V$ over $O$ (that is, with a minimum number of critical cells), the cell pairings are disjoint pairs of incident cells. In this case, the connected directed graphs (DG for short) of the HSF can be described by the union of different non-trivial closed $V$-paths [8]. 

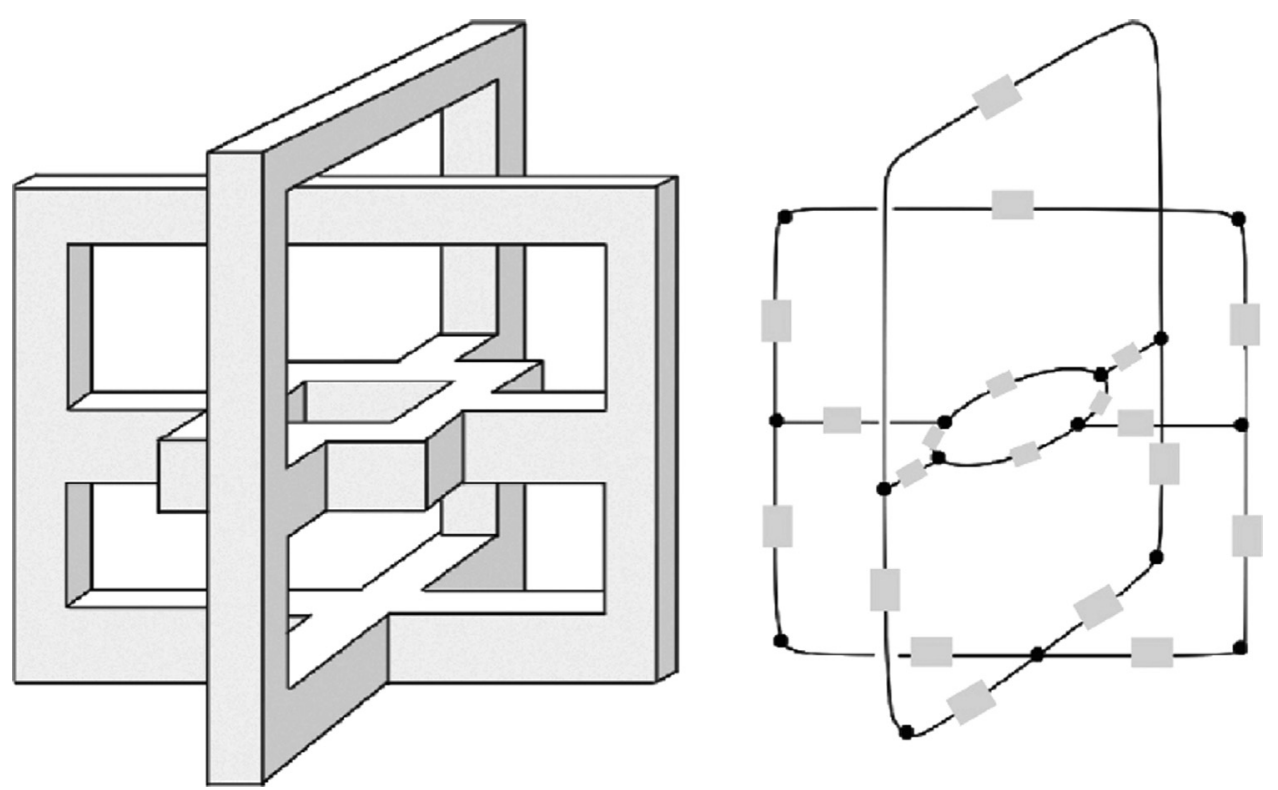

Fig. 2. A polyhedron shown by [15] and a homotopically equivalent cell complex extending Fig. 6.19 of [13].

For doing so, a chain contraction is defined by a homology chain integral operator $\phi_{F}$ (homology $\chi$ operator, h $\chi$ ), which maps an $i$-cell into a linear combination of $(i+1)$-cells. The following examples provided an introduction for $\phi_{F}$ and its associated HSF. In both examples, we construct HSF DG structures from optimal discrete gradient vector fields.

The linear operator $\phi_{\mathcal{F}}$ maps an $i$-cell into a linear combination of $(i+1)$-cells and satisfies certain conditions, to be formally defined later in Definition $1 .{ }^{1}$ The map $\phi_{\mathcal{F}}$ is specified by an HSF $\mathcal{F}$. The following examples provide an introduction for $\mathrm{h} \chi$ map and its associated HSF, and the formal definitions in the next section.

Example 1. Consider the object in Fig. 2 (left) and one homotopically equivalent cell complex $\mathcal{X}$ that is the graph shown in Fig. 2 (right). Each dot in the graph represents a 0-cell, and each grey rectangle represents a 1-cell. Frontiers are directly defined for every 1-cell as the sum of its two endpoints.

Considering the connected 1-dimensional cell complex in Fig. 2 (right), the construction of its HSF representation can be done in a straightforward manner. The HSF in this case consists of a single tree spanning every 0 -cell in the graph, and a maximal pairing between 0 -cells and 1-cells defined in this tree (minimizing to one the number of critical cells at dimension zero).

Fig. 3 shows a (non-unique) HSF $\mathcal{F}_{1}$ associated with the cell complex in Fig. 2 (right). Each arrow illustrates a cell pairing from a 0 -cell to a 1-cell. We can observe that every 0 -cell is paired with a 1-cell except the 0 -cell $j$ that is not paired. Six 1-cells also remain unpaired (shown in light grey). Unpaired cells are called critical cells, and each one of them corresponds to one homology class. In general, in an HSF derived from an optimal discrete gradient vector field, the number of unpaired $0-, 1-$, and 2-cells coincides with the Betti numbers $\beta_{0}, \beta_{1}$, and $\beta_{2}$, respectively. In this example, we have one connected component $\left(\beta_{0}=1\right)$ and six cycles $\left(\beta_{1}=6\right)$, that is one unpaired 0-cell and six unpaired 1-cells.

The $\mathrm{h} \chi$ map $\phi_{1}$, associated with HSF $\mathcal{F}_{1}$, is defined (in this case of a 1-dimensional complex) only as the sum of 1-cells that are in

\footnotetext{
${ }^{1}$ For cell complexes and linear combinations of cells, see, for example, Chapter 6 in [13]. For constructing the map $\phi_{\mathcal{F}}$, see also the papers $[10,9]$ and $[20]$ in the cubical setting.
}

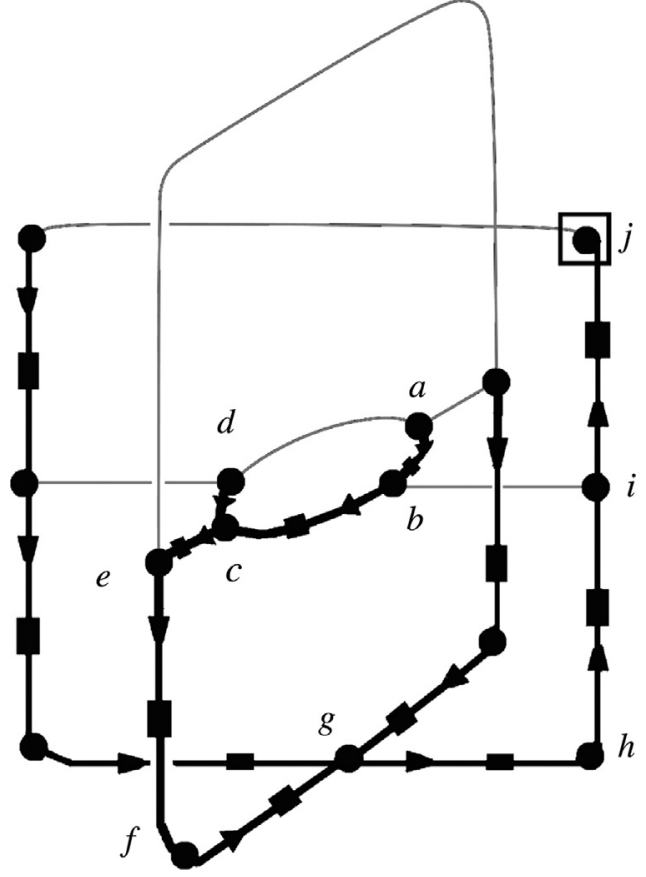

Fig. 3. HSF $\mathcal{F}_{1}$ of the example shown in Fig. 2.

the path from any vertex to a critical cell $j$. For example,

$\phi_{1}(a)=a b+b c+c e+e f+f g+g h+h i+i j$ and

$\phi_{1}(g)=g h+h i+i j$

where the 1-cell $a b$ is the one with endpoints $a$ and $b, b c$ with $b$ and $c$, and so forth.

Example 2. Consider a hollow tetrahedron $\mathcal{X}$ as a simple example of a cell complex; it has one connected component and one cavity.

In Fig. 4 (left), 0-cells, 1-cells, and 2-cells are again represented by black dots, grey rectangles, and grey dots, respectively. Geometrically, locations of those symbols may be identified with centroids of cells. For example, the frontier of the 2-cell acd is 

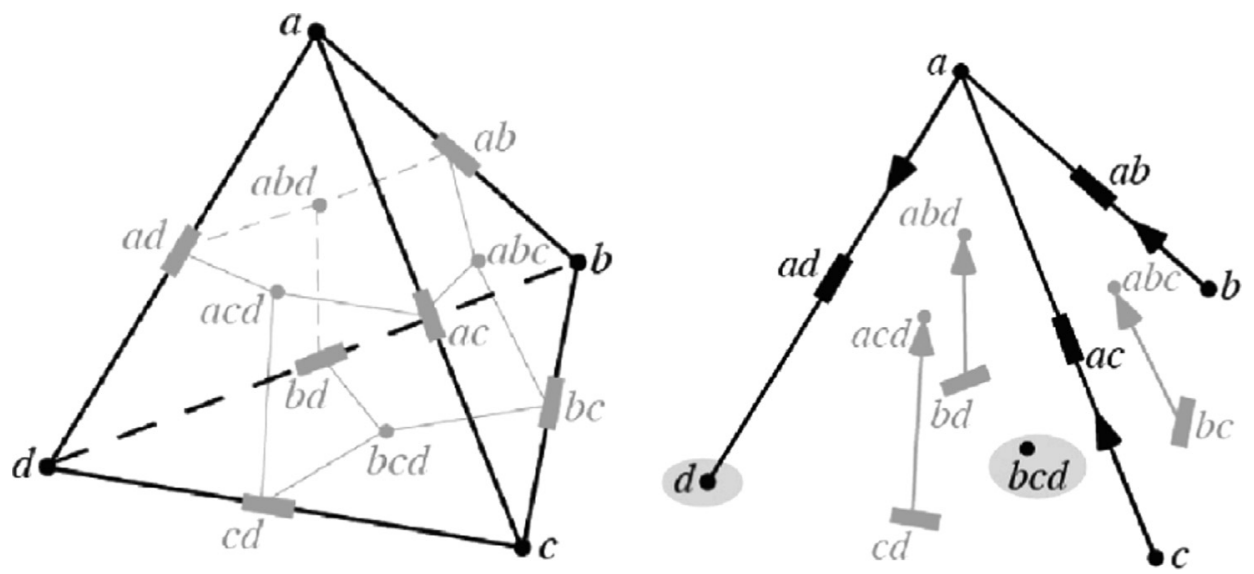

Fig. 4. Left: Visualization of a cell complex of a hollow tetrahedron. Right: HSF $\mathcal{F}_{1}$ of a hollow tetrahedron; cells $d$ and $b c d$ remain unpaired.

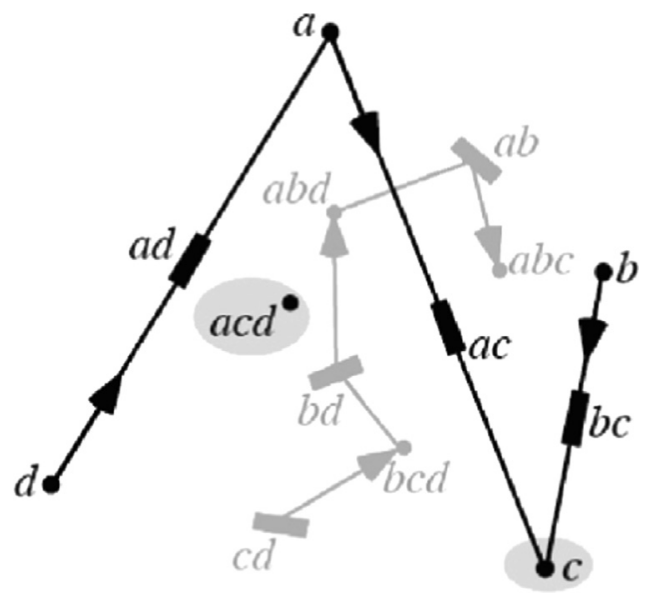

Fig. 5. HSF $\mathcal{F}_{2}$ of a of a hollow tetrahedron. Cells $c$ and acd remain unpaired.

specified by $\vartheta(a c d)=a c+a d+c d$; cells $a, c, d$ are incident with cell acd, and cell acd is incident with cells $a c, a d$, and $c d$.

Fig. 4 (left) shows an undirected graph $\mathcal{G}(X)$ defined by nodes (i. e. the different cells) of $\mathcal{X}$ and incidences between an $i$-cell and an $(i+1)$-cell. Figs. 4 (right) and 5 show two different HSFs $\mathcal{F}_{1}$ and $\mathcal{F}_{2}$ for $\mathcal{X}$, being directed subgraphs of $\mathcal{G}(X)$. As mentioned before, an arrow on an edge illustrates a cell pairing from an $i$-cell to an $(i+1)$-cell.

In Fig. 4 (right), a black (0,1)-tree connects all the 0 -cells with three of the 1-cells. Pairings between the remaining three 1-cells and incident 2-cells define three (1,2)-trees (in grey) having only two nodes each. Finally, there is one unique HSF tree (also in black) formed by the 2-cell bcd only. This finalizes a spanning forest $\mathcal{F}_{1}$. Every 0 -cell is paired with a 1-cell (pairs are $(a, a d),(c, a c)$ and $(b$, $a b)$ ) except the 0 -cell $d$ which is not paired. Every 1 -cell is paired, either with a 0 -cell (in black) or a 2-cell (in grey). Regarding the 2cells, only bcd remains unpaired. The only unpaired cells in Fig. 4 (right) are $d$ and $b c d$, corresponding with the Betti numbers $\beta_{0}=1$ and $\beta_{2}=1$.

Any 0-cell of the tetrahedron can be selected to become the critical cell. In Fig. 5, cell $c$ is the critical cell for the $(0,1)$-tree. The $(1,2)$-tree (in grey) has no critical cell (and $a b c$ as a root). The cell acd is a single-node tree. The shown HSF $\mathcal{F}_{2}$ consists of three trees and two critical cells $c$ and acd.

The h $\chi$ operator $\phi_{1}$, associated with $\operatorname{HSF} \mathcal{F}_{1}$, is defined as follows:

$$
\begin{array}{ll}
\phi_{1}(a)=a d, & \phi_{1}(b)=a b+a d, \\
\phi_{1}(c)=a c+a d, & \phi_{1}(d)=0(\text { critical cell }),
\end{array}
$$

$$
\begin{array}{ll}
\phi_{1}(a b)=0, & \phi_{1}(a c)=0 \\
\phi_{1}(a d)=0, & \phi_{1}(b c)=a b c, \\
\phi_{1}(b d)=a b d, & \phi_{1}(c d)=a c d, \\
\phi_{1}(a b c)=0, & \phi_{1}(a b d)=0, \\
\phi_{1}(a c d)=0, & \phi_{1}(b c d)=0 \text { (critical cell). }
\end{array}
$$

The h $\chi$ map $\phi_{2}$ associated with HSF $\mathcal{F}_{2}$ is as follows:

$$
\begin{aligned}
& \phi_{2}(a)=a c, \\
& \phi_{2}(c)=0 \text { (critical cell), } \\
& \phi_{2}(a b)=a b c, \\
& \phi_{2}(a d)=0, \\
& \phi_{2}(b d)=a b d+a b c, \\
& \phi_{2}(a b c)=0, \\
& \phi_{2}(b c d)=0,
\end{aligned}
$$

$$
\begin{aligned}
& \phi_{2}(b)=b c, \\
& \phi_{2}(d)=a d+a c, \\
& \phi_{2}(a c)=0, \\
& \phi_{2}(b c)=0, \\
& \phi_{2}(c d)=b c d+a b d+a b c, \\
& \phi_{2}(a b d)=0, \\
& \phi_{2}(a c d)=0 \text { (critical cell). }
\end{aligned}
$$

This concludes the example, where two possible HSF representations for the tetrahedron are shown. Notice that the critical cells are the ones that remain unpaired.

The map $\phi_{\mathcal{F}}$ determines a way in which the cell complex is algebraically reduced into its homological minimal expression. It may be understood as "a kind of" an inverse of the algebraic frontier of the cell complex 0 . The algebraic frontier is denoted by $\vartheta O$ and also known as chain frontier (see Section 5.2 in [13]); the chain frontier decreases the dimension of complex $O$ by one; $\vartheta$ is a linear operator which also satisfies that $\vartheta \vartheta O=0$.

Definition 1. A function $\phi_{\mathcal{F}}$, called $\mathrm{h} \chi$ operator, is a linear map

$\phi_{\mathcal{F}}: C_{*}(\mathcal{O}) \rightarrow C_{*+1}(\mathcal{O})$

satisfying the following identities:

(a) $\phi_{\mathcal{F}} \phi_{\mathcal{F}}=0$, called 2-nilpotency,

(b) $\phi_{\mathcal{F}} \vartheta \phi_{\mathcal{F}}=\phi_{\mathcal{F}}$, the splitting homotopy condition, and

(c) $\vartheta \phi_{\mathcal{F}} \vartheta=\vartheta$, known as splitting homology condition.

The algebraic definition of an $\mathrm{h} \chi$ map follows "naturally" from the notion of "splitting homotopy" in [1]. Functions $\phi_{1}$ and $\phi_{2}$, deduced from their corresponding combinatorial HSF structures, satisfy conditions (a), (b), and (c) of an h $\chi$ map.

The combinatorial scaffolding HSF codifies (in an economical way, and without redundancies) the values of the $\mathrm{h} \chi$ map, specifying the number of $\mathcal{V}$-paths from an $i$-cell to any of its associated homological critical cells.

In this paper, we visualize an HSF $\mathcal{F}$, but mathematically we handle its canonically associated chain-homotopy $\phi_{\mathcal{F}}$. 
For further details, we refer to $[17,18]$ on HSFs, and to $[10,9]$ and [20] on determining an $\mathrm{h} \chi$ map.

\section{Fractal polyhedra}

Now consider a fractal polyhedron $\mathcal{O}$ as being a convex cell complex. Starting from an optimal discrete gradient vector field $V$ we build an HSF structure for $\mathcal{O}$, and, consequently, an h $\chi$ map. Any $(i, i+1)-D G$ of this complex is a directed subgraph of the undirected graph $\mathcal{G}(O)$ having as nodes the convex cells of the complex, and edges that represent incidences between $i$ - and $(i+1)$-cells.

A $(0,1)$-tree $T^{0}$ spans all the 0 -cells of a connected component of $\mathcal{G}(0)$ via intermediate 1 -cells in $\mathcal{G}(0)$.

These intermediate edges in $T^{0}$ are labelled by arrows going from a 0 -cell to a 1-cell, defining a pairing, and specifying in this way the starting optimal gradient vector field $V$ at this level (i.e., the number of critical cells is minimal, and thus equal to the corresponding Betti number).

The set of $(0,1)$-trees (in case there is more than one connected component) is denoted by $\mathcal{F}^{0}$. Let us suppose that we have determined the set $\mathcal{F}^{i-1}$ of $(i, i+1)$-DG.

A connected $(i, i+1)$-DG $T^{i}$ is a directed graph spanning every $i$-cell of a connected component of $\mathcal{G}(O) \backslash\left(\mathcal{F}^{0} \cup \mathcal{F}^{i-1}\right)$ such that a maximal number of $i$-cells is connected with adjacent $(i+1)$-cells in $T^{i}$. The cell pairings in this connected direct subgraph are determined by those in the vector field $V$.

The number of non-paired $i$-cells of $T_{i}$ (i.e. of critical cells) is called the criticality number of the DG $T^{i}$.

The set of $(i, i+1)$-DGs is denoted by $\mathcal{F}^{i}$. The criticality of $\mathcal{F}^{i}$ (also called the $i$-criticality of the associated HSF) is the sum of criticalities of the graphs of $\mathcal{F}^{i}$. An HSF $\mathcal{F}$ of a fractal set $\mathcal{O}$ satisfies that its $i$-criticality coincides with the corresponding $i^{\text {th }}$ Betti number of $\mathcal{O}$.

As commented above, starting from an HSF structure of a fractal and fixing a field of coefficients, one can determine an algebraic relationship between the differential graded vector field of linear combinations of cells belonging to the fractal, denoted by $C_{*}(\mathcal{O})$, and its homology graded vector space. The latter one is a homology chain contraction of $\mathcal{O}$.

All the DGs of the HSF structure describe "connected" versions of the optimal discrete gradient vector field $V$ at different levels, and a $h \chi$ map can be directly constructed.
We define a linear mapping

$\phi: C_{*}(\mathcal{O}) \rightarrow C_{*+1}(\mathcal{O})$

by $\phi(x)=(\sigma \bmod 2) Y$, for all the $(i+1)$-cells $Y$ such that there are exactly $\sigma$ paths from $x$ to $Y$ through an connected $(i, i+1)$-DG of the HSF structure. Obviously, $\phi$ applied to a critical cell is equal to zero. The stated three properties of 2-nilpotency, splitting homotopy and splitting homology allow us to use $\phi$ for performing cycle calculus. Topological analysis of, and cycle calculus on fractals can be simplified by using the spanning HSF forests.

In the following two sections we apply HSF forests for developing cycle calculus on the Menger sponge and two versions of Sierpiński pyramids.

\section{Menger sponge}

The Menger sponge has been illustrated already in Fig. 1. In general, $\mathcal{M}_{n}$ denotes the $n$ th-recursion Menger sponge. For example, Fig. 6 shows on the left the 1st-recursion Menger sponge $\mathcal{M}_{1}$. If considered as a solid polyhedron then it is formed by 20 cubical 3-cells.

In this section, an HSF combinatorial scaffolding for the Menger sponge is constructed "ad hoc", based on a tree-cotree strategy. The HSF is a subgraph of the simplicial graph $\mathcal{G}(0)$, which we construct as a barycentric subdivision of a regular cubical cell complex. Fig. 6 shows on the right (in red) the (0,1)-tree of an HSF of this set, where crosses show 0 -cells of $\mathcal{G}(O)$, diamonds 1 -cells, and a critical cell is encircled at the lower left. This tree (shown in red) already determines the single connected component of the Menger sponge. An arrow on an edge of the (0,1)-tree specifies a disjoint pairing between a 0-cell and a 1-cell. In this case, the set of arrows determines an optimal discrete gradient vector field for this $(0,1)$ level.

Fig. 7 shows two of the four (1,2)-trees having a non-zero criticality number. These two trees have a criticality number equal to one or two. The 2-cells are drawn using hollow 5-pointed stars. The other two (1,2)-trees have zero criticality. These four $(1,2)-$ trees of $\mathcal{M}_{1}$ determine the different 1 -cycles generating the first homology group of this 1st-recursion Menger sponge, or dually, the different cocycles generating its first cohomology group.

Finally, Fig. 8 shows the different (2,3)-trees of the HSF of $\mathcal{M}_{1}$, all of them having zero as criticality number. Starting from the (0,1)-tree and the (2,3)-trees (cotrees), all the (1,2)-trees of the HSF structures are completely defined. This tree-like coding of $\mathcal{M}_{1}$
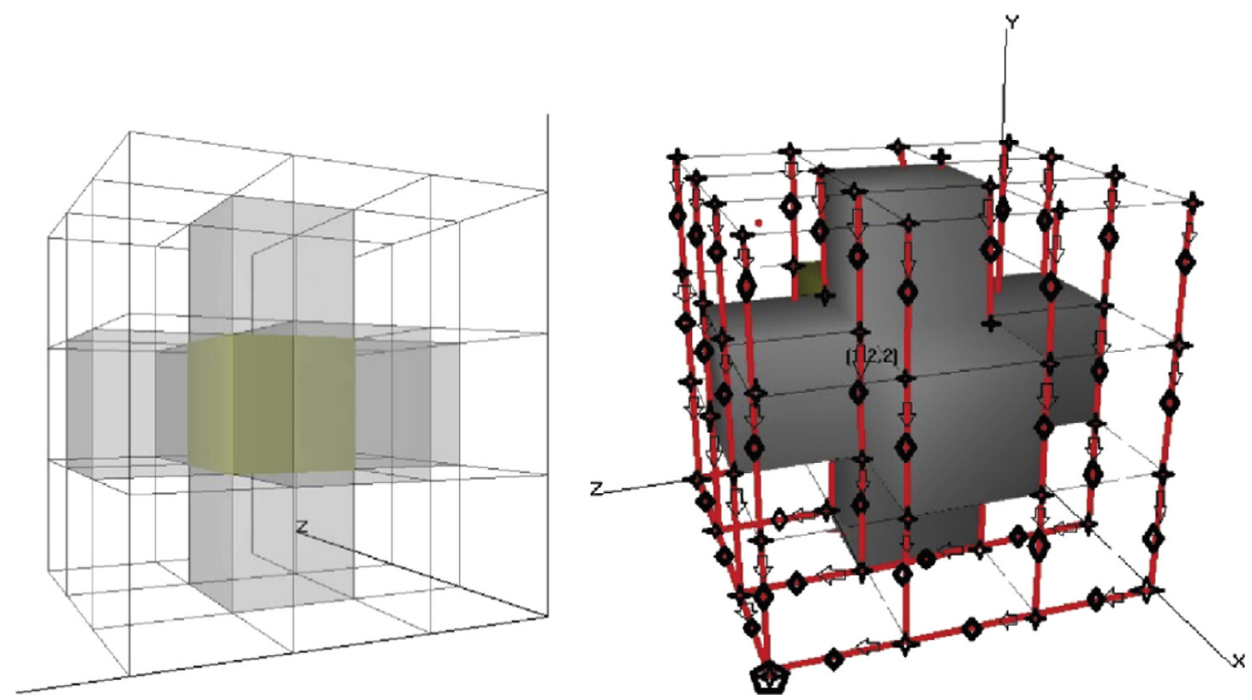

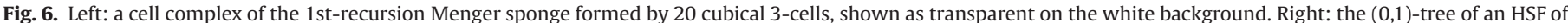
this cell complex (in red). (For interpretation of the references to color in this figure caption, the reader is referred to the web version of this paper.) 

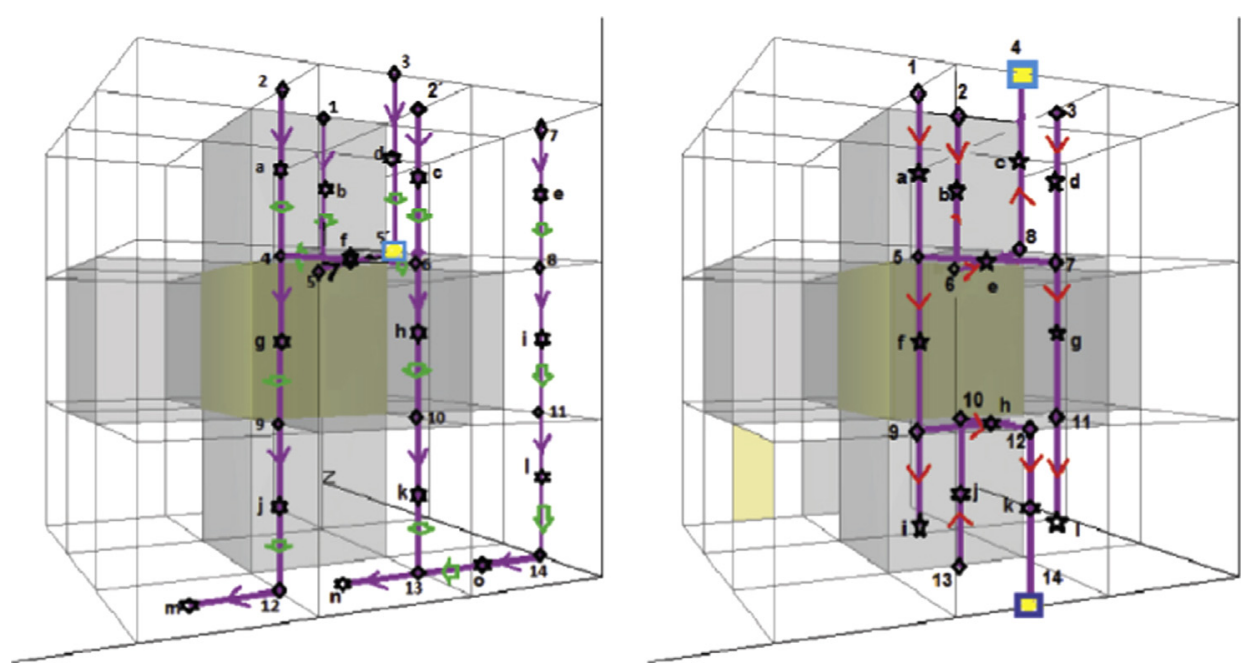

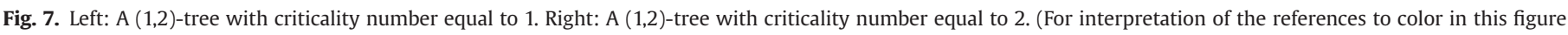
caption, the reader is referred to the web version of this paper.)

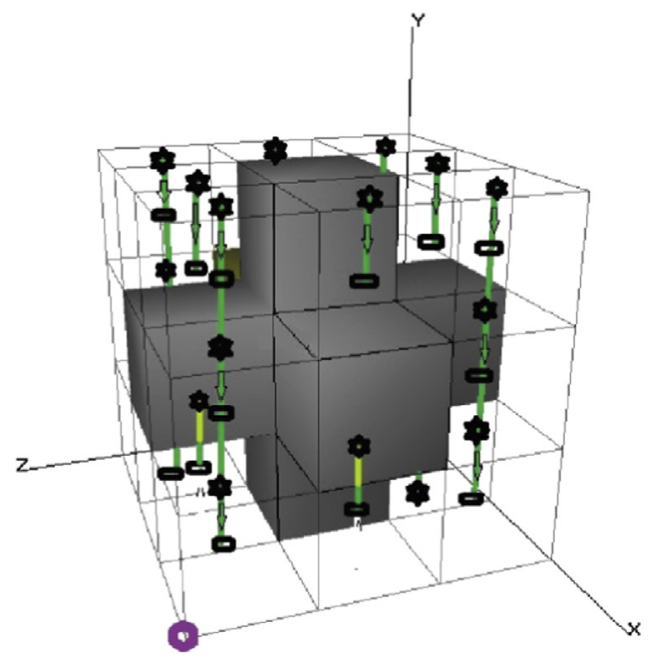

Fig. 8. (2,3)-trees of the HSF of $\mathcal{M}_{1}$, all of criticality zero.

provides now a way, as specified earlier, to determine its associated h $\chi$ map $\phi$.

For example, for the (1,2)-tree $T$ shown in Fig. 7 on the left, with criticality number one, it is straightforward to determine its associated $\mathrm{h} \chi$ map $\phi$. This is because it specifies a true optimal discrete gradient vector field. The critical 1-cell of this tree (see the pale blue square) is indexed in the Fig. by " 5 ".

We refer to the indices of cells $1,2,2^{\prime}, 3, \ldots, 14$ as specified on the left of Fig. 7. First, we derive a map $\phi^{\prime}$ defined by:

$$
\begin{aligned}
& \phi^{\prime}(1)=b, \\
& \phi^{\prime}\left(2^{\prime}\right)=c+h+k+n, \\
& \phi^{\prime}(4)=g+j+m, \\
& \phi^{\prime}\left(5^{\prime}\right)=f+g+h+j+k+m+n, \\
& \phi^{\prime}(7)=e+i+l+o+n, \\
& \phi^{\prime}(9)=j+m, \\
& \phi^{\prime}(11)=l+o+n, \\
& \phi^{\prime}(13)=n,
\end{aligned}
$$

$$
\begin{aligned}
& \phi^{\prime}(2)=a+g+j+m, \\
& \phi^{\prime}(3)=d+f \\
& \phi^{\prime}(5)=0 \text { (critical cell), } \\
& \phi^{\prime}(6)=h+k+n \\
& \phi^{\prime}(8)=i+l+o+n, \\
& \phi^{\prime}(10)=k+n \\
& \phi^{\prime}(12)=m \\
& \phi^{\prime}(14)=0+n .
\end{aligned}
$$

For any 2-cell in $T$, the value of $\phi^{\prime}$ is 0 . It is also zero for any other cell of the cell complex. Then, the following equalities are valid:

$\phi^{\prime} \phi^{\prime}=0, \quad \phi^{\prime} \vartheta^{\prime} \phi^{\prime}=\phi^{\prime}, \quad$ and $\quad \vartheta^{\prime} \phi^{\prime} \vartheta^{\prime}=\vartheta^{\prime}$ where $\vartheta^{\prime}$ is the map that assigns to any 2-cell $\alpha$ in $T$ the sum of 1cells in the tree that belong to the frontier of cell $\alpha$.

The final $\mathrm{h} \chi$ map $\phi$ is defined as follows for the cells in $T$ :

$$
\begin{array}{ll}
\phi^{\prime}\left(2^{\prime}\right)=c+h+k+n+m, & \phi^{\prime}(5)=0 \text { (critical cell), } \\
\phi^{\prime}\left(5^{\prime}\right)=f+g+h+j+k+n, & \phi^{\prime}(6)=h+k+n+m, \\
\phi^{\prime}(7)=e+i+l+o+n+m, & \phi^{\prime}(8)=i+l+o+n+m, \\
\phi^{\prime}(10)=k+n+m, & \phi^{\prime}(11)=l+o+n+m, \\
\phi(13)=n+m, & \phi^{\prime}(14)=0+n+m,
\end{array}
$$$$
\phi=\phi^{\prime} \text { otherwise. }
$$

It is straightforward to verify the validity of 2-nilpotency, splitting homotopy and splitting homology conditions for $\phi$ and the real frontier of the original cell complex.

An important aspect is that the constructed HSF structure should have, if possible at all, their critical cells on the "external" frontier of the cell complex, in order to determine more efficiently whether a considered cycle is either "created" or "destroyed" when we add copies of 0 -recursion order Menger sponges to a given complex.

In order to determine the Betti numbers of polyhedron $\mathcal{M}$, we take into consideration:

1. a simplified graph description of Menger sponges in order to easily handle HSF structures,

2. the fact that the number of critical cells needs to be minimal, and

3. that these critical cells need to be located in symmetric positions at "intersections" of Menger sponges of lower dimensions.

In algebraic-topological terms and within this setting, we apply here a kind of constructive Mayer-Vietoris technique for involved HSF structures and, subsequently, for the corresponding $\mathrm{h} \chi$ maps. In this way, we obtain the following recursion equations:

Theorem 1. For the number $T_{n}$ of tunnels of the nth-recursion polyhedral Menger Sponge $\mathcal{M}_{n}$, we have that

$T_{1}=5$

$T_{n}=8 \cdot T_{n-1}+\left[T_{n-1}-2 \cdot\left(8^{n-2}+8^{n-3}+\ldots+8+1\right)\right] \cdot 12+5$ 
$T_{n}=20 \cdot T_{n-1}-24 \cdot\left(8^{n-2}+8^{n-3}+\ldots+8+1\right)+5$

Proof. Let us consider a description of the different levels of the Menger sponge in terms of graphs (i.e. 1-dimensional cell complexes). For instance, the 1-dimensional Menger sponge is a hollow cube. The $n$ th-recursion polyhedral Menger sponge $\mathcal{M}_{n}$ is obtained from the $(n-1)^{\text {th }}$-recursion Menger sponge $\mathcal{M}_{n-1}$ by simply considering a $3 \times 3 \times 3$ cube (that is 27 voxels) in which each of the eight corner voxels, and each of its side voxels (there are four in each face, and they are 6-adjacent to the corner voxels) is a $\mathcal{M}_{n-1}$ Menger sponge. The central voxel of each face, and the central voxel of the cube are void. See Fig. 9.

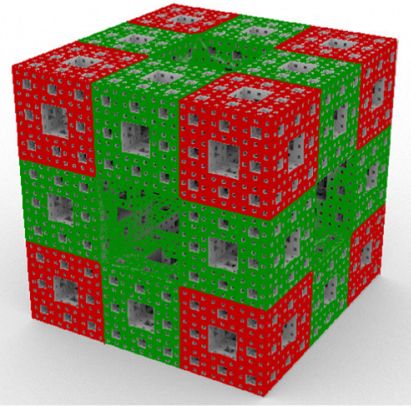

Fig. 9. Corner voxels colored in red, and side voxels coloured in green. (For interpretation of the references to color in this figure caption, the reader is referred to the web version of this paper.)

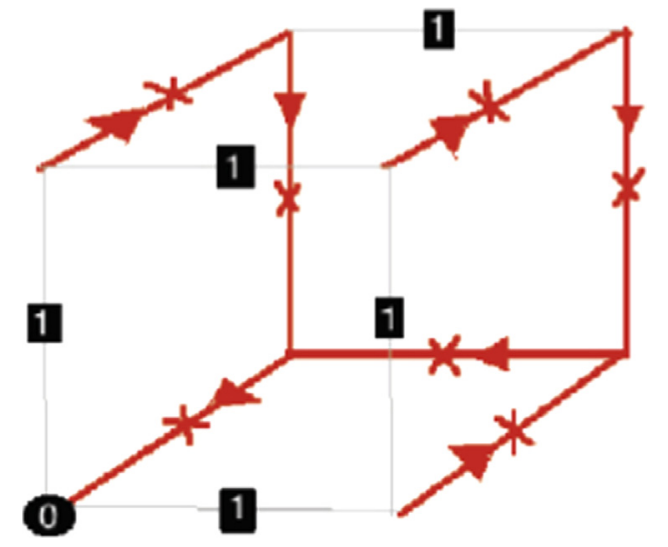

Fig. 10. Graph-skeleton of the 1-recursion polyhedral Menger sponge.
Depicting the 1-recursion polyhedral Menger sponge by means of a graph-skeleton, we choose the HSF structure presented in Fig. 10 .

There is a $(0,1)$-HSF tree (drawn in red) showing that there is one connected component (one critical cell drawn by a black filled small circle) and five 1-2 trivial HSF graphs (five 1-holes whose associated critical cells are drawn by black filled small squares). A h $\chi$ map can be straightforwardly constructed from the previous HSF.

In the $n=1$ case, the graph that represents $\mathcal{M}_{1}$ has $2 \times 2 \times 2=8^{1}$ vertices. For $n=2$ we introduce a $\mathcal{M}_{1}$ Menger sponge in each of the corner and side voxels. Therefore, the final $\mathcal{M}_{2}$ graph has $(3+1) \times(3+1) \times(3+1)=4 \times 4 \times 4$ vertices, that is $2^{6}=8^{2}$. In Fig. 11, corner voxels are represented by a light blue square, and side voxels by a green circle. The way in which the $\mathcal{M}_{1}$ HSFs are connected is also shown. The number of edges that need to be added are 7 (in red) in order to generate the $\mathcal{M}_{2}$ HSF (three in the frontal face, three in the back face, and one more in the middle face that connects both faces).

This HSF specifies $T_{2}$. In the unit $3 \times 3 \times 3$ cube, each corner voxel contributes with $T_{1}$ (equal 5 ) holes, each of the 12 side voxels contributes with $T_{1}-2 x(1)$ holes and, finally, the central voxel contributes with five holes (i.e. 12 edges of this central voxel minus seven edges from the (0-1)-HSF tree). Summing up, there are 81 holes for $M_{2}$.

For the general case $n \geq 2$, we only need to show that the minimal number of edges $a_{f(n)}$ in a 2-dimensional face of the cube, containing an $n$-dimensional Menger sponge belonging to any HSF,

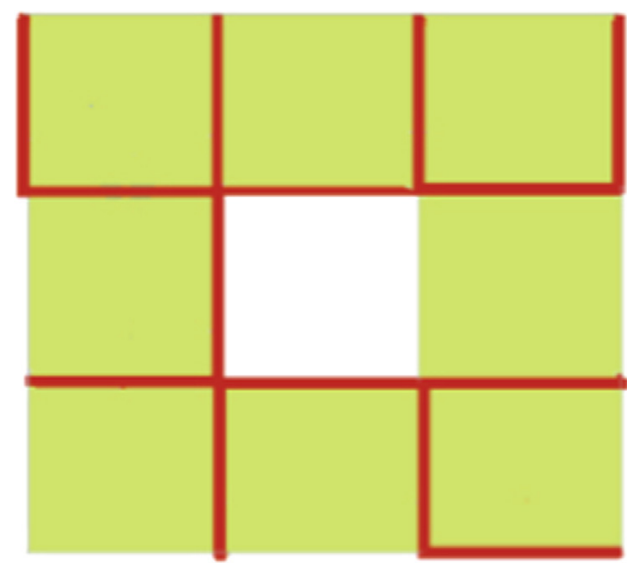

Fig. 12. Colored in red the edges belonging to one HSF in one face of the cube $3 \times 3 \times 3$. (For interpretation of the references to color in this figure caption, the reader is referred to the web version of this paper.)
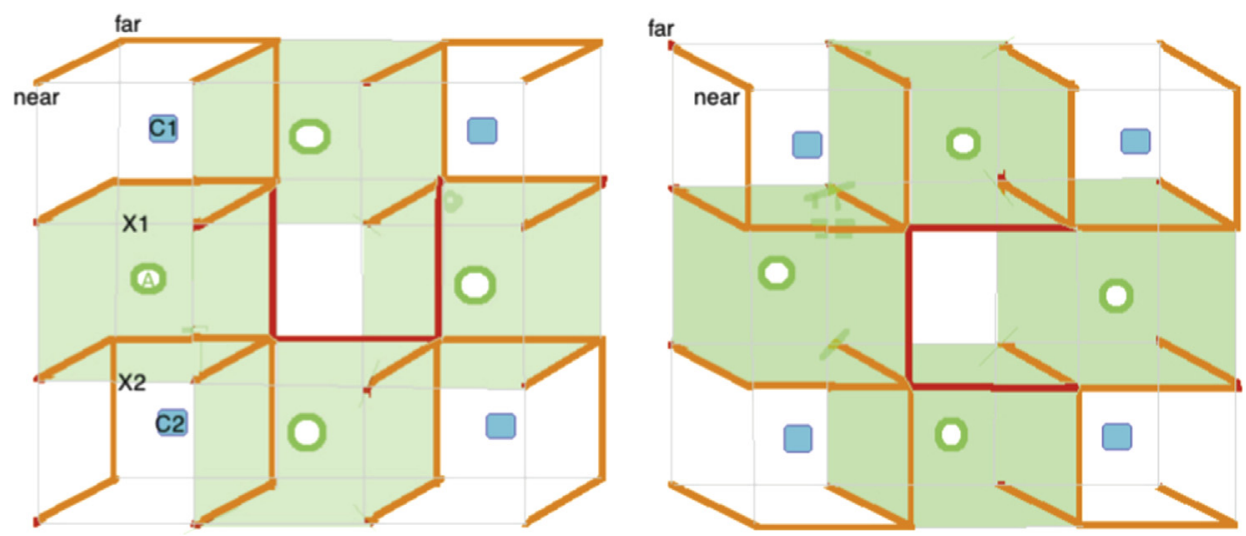

Fig. 11. Graph-skeleton of the frontal and back face of the 2-recursion polyhedral Menger sponge. 
is equal to $8^{(n-1)}+\ldots+8^{1}+1$. These edges can be seen as $1-2$ HSF trivial trees. This fact can be easily proven taking into account that there are faces of eight voxels $M_{n-1}$ in each 2-dimensional face of the unit $3 \times 3 \times 3$ cube $C$, using HSF structures of this face, like the one shown in Fig. 12. It is straightforward to show that $a_{f(n)}$ is $8 \times a_{f(n-1)}+1$, being the last summand for the contribution of the empty central voxel of this face.

In fact, we do not need to specify any HSF in order to count the number of holes for the graph complex $M_{2}$ or, more generally, $M_{n}$. $T_{n}$ equals $e_{n}-v_{n-1}$, where $v_{n}$ is the number of vertices, and $e_{n}$ is the number of edges in the $n$-dimensional Menger sponge.

As an example for Theorem 1, we have that $T_{2}=81$ and $T_{3}=1409$. Note that the number of tunnels (i.e. the Betti number $\beta_{1}$ of the $n$ th-recursion Menger cube) increases at a very fast rate.

Concerning the cycle calculus, due to the fact that we can compute an explicit $\mathrm{h} \chi$ map $\phi_{n}$ for any $n$ th-recursion Menger sponge, it is possible to classify any cycle within the fractal (specifying its homology class), or to determine whether two cycles within the fractal polyhedron are homologous or not (that is, if they can be, or cannot be transformed one into the other in a continuous way within the Menger sponge).

\section{Sierpiński pyramid}

At first we consider the Sierpiński square-based pyramid $S_{0}$; see Fig. 13 for an example at some iteration step. This pyramid generalizes the Sierpinski sieve (see [26]) into 3D space. The recursion starts with one square pyramid. At Level $n+1$, five copies of $S_{n}$ (i.e., pyramids at Level $n$ ) form the pyramid $S_{n+1}$, where four copies of $S_{n}$ form its base, and the fifth copy sits on the four top-vertices of those four copies.

In this case, using the HSF-technique for this fractal set, it follows straightforwardly how to determine a formula for the number of tunnels of the polyhedron $\mathcal{S}_{n}$ :

Theorem 2. For the nth-level Sierpinski square-based pyramid, we have that

$T_{1}=3$

$T_{n}=3 \cdot\left(5^{n-1}+5^{n-2}+\ldots+5^{1}+5^{0}\right)$

Proof. A HSF-structure of the recursion-0 Sierpiński square-based pyramid $S_{0}$ is shown in Fig. 14, left.

In order to construct the HSF for the first recursion Sierpiński pyramid, let us consider the cellular structure as shown in Fig. 14 (right).

In Fig. 14 (right), the four squares coloured in light blue are the squared basis of five $S_{0}$ Sierpiński pyramids. We consider that each of these five pyramids has the HSF represented in Fig. 14 (right). The five squared cells coloured in yellow, the twelve dark grey edges, and the vertices marked with a circle are the sinks of the HSF trees in $S_{0}$, and will be eliminated when applying the $\mathrm{h} \chi$ operations in the construction of $S_{1}$.

In Fig. 15 (left), the $\mathrm{h} \chi$ operations corresponding to the $0-1 \mathrm{HSF}$ of the five copies of $S_{0}$ are coloured in red. After applying these reductions, each of the four vertices in the $S_{0}$ bases will be contracted to a single vertex (marked by a circle in Fig. 15, left).
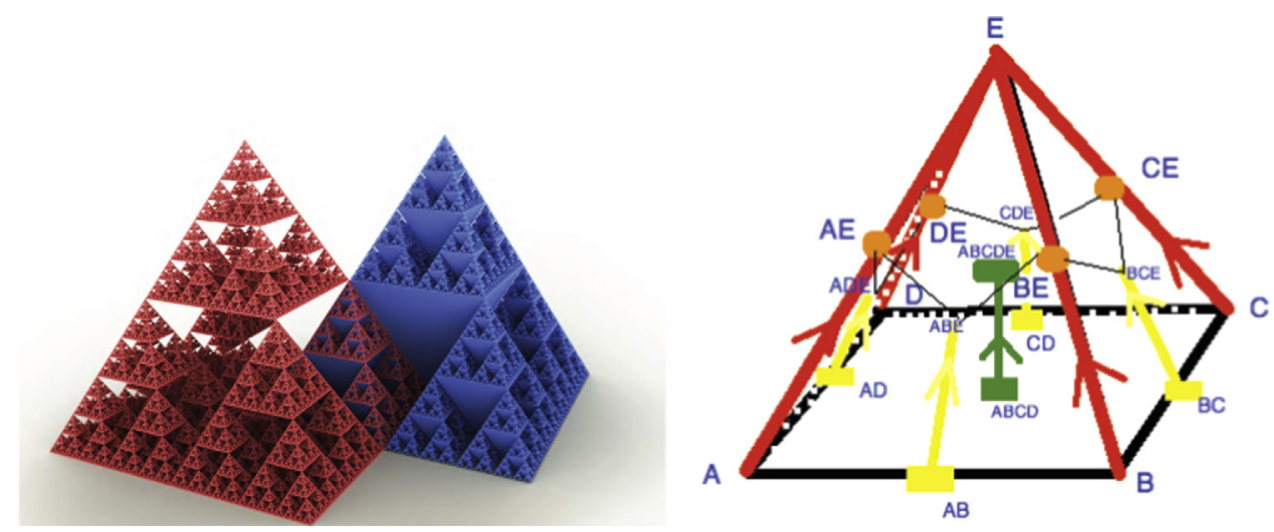

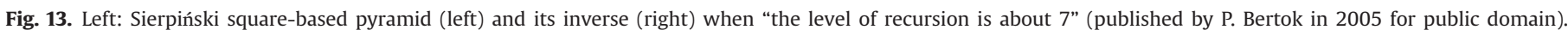
Right: HSF structure for the square-based pyramid $S_{0}$.
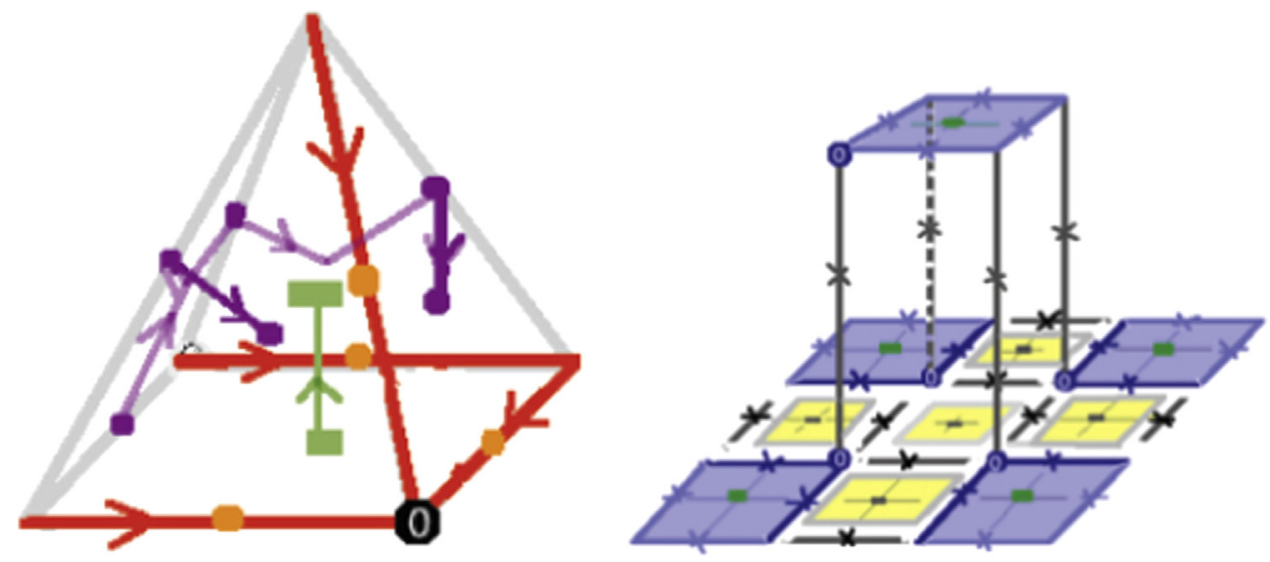

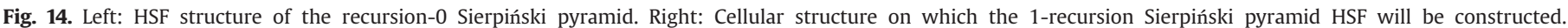
(For interpretation of the references to color in this figure caption, the reader is referred to the web version of this paper.) 

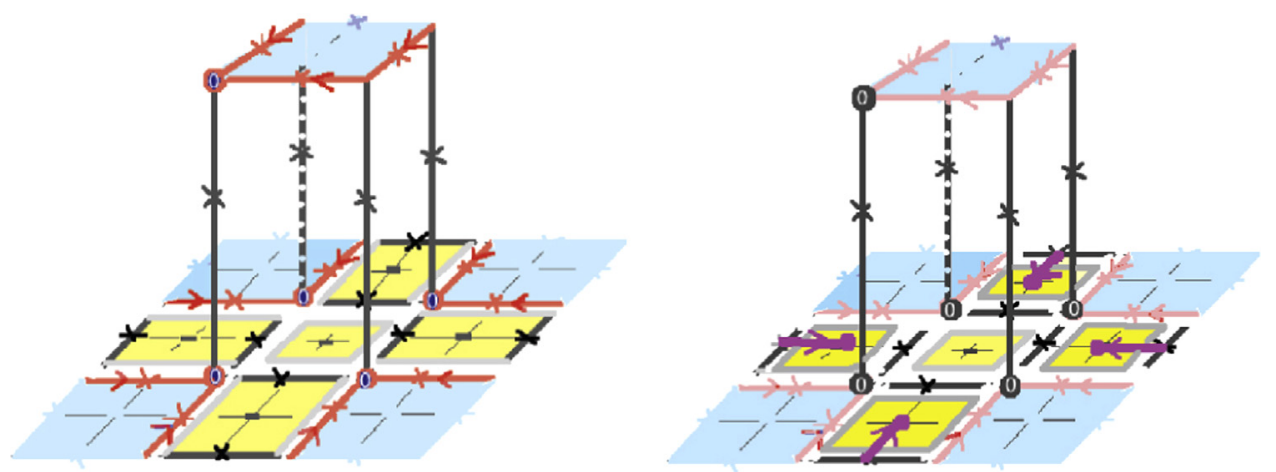

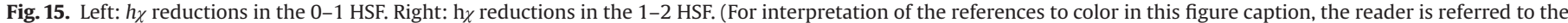
web version of this paper.)

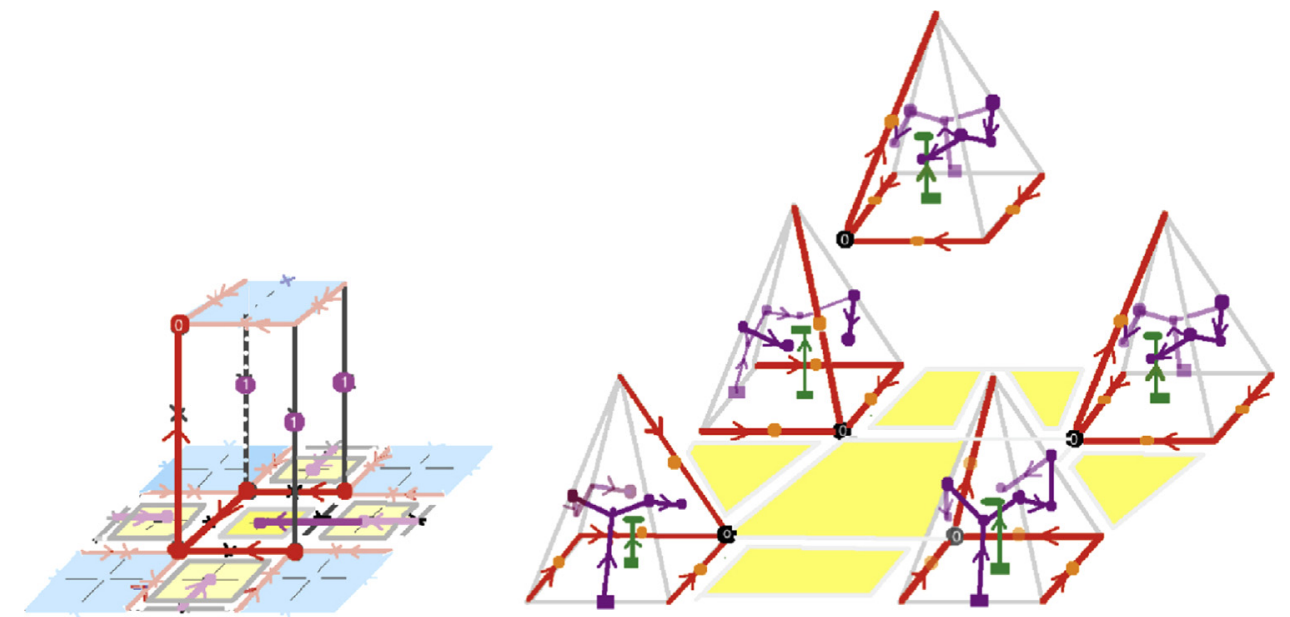

Fig. 16. Left: Remaining h $\chi$ reductions for the construction of $S_{1}$. HSF of $S_{1}$.

Therefore, each of the $S_{0}$ pyramids contributes with a single critic cell in dimension zero (that is, one connected component for each $S_{0}$ pyramid).

In Fig. 15 (right), the $\mathrm{h} \chi$ operations in 1-2 colored in purple represent the elimination of the yellow squared cells.

After the previously mentioned operations, let us consider the HSF of the remaining cells shown in Fig. 16 (left). From this final structure we can deduce that $S_{1}$ has one connected component (marked by a 1 ) and three 1-dimensional holes (marked by a 0 ). Note that the lower extreme of the vertical edges corresponds in reality to the four $S_{0}$ apexes, but they are shown here in the corner for visualization purposes.

In a similar way, we can compute the number of tunnels for higher levels of recursion, where we have that

$T_{n}=5 \cdot T_{n-1}+3$

In Fig. 16 (right), an HSF (without taking into account the initial h $\chi$ operations) for the first level of the recursive Sierpiński pyramid is shown.

As an example for Theorem 2, $T_{1}=3$ is followed by $T_{2}=18$, and then $T_{3}=93$. In fact, the HSF-structure for the $n$-level Sierpiński square-based pyramid can be derived almost automatically from that of level 0; see Fig. 13.

From the HSF-structure of $\mathcal{S}_{n}$ and, more precisely, from the associated $\mathrm{h} \chi$ map, it is possible to derive a cycle calculus for these fractal polyhedra. If the fractal polyhedron is the Sierpiński triangle-based pyramid, then we have the following:
Theorem 3. For the nth-level Sierpinski triangle-based pyramid, we have that

$T_{1}=3$

$T_{n}=3 \cdot\left(4^{n-1}+\ldots+4^{1}+4^{0}\right)$

Theorem 3 follows analogously to Theorem 2 .

\section{Experiments}

Our experiments illustrate how to apply the proposed techniques to mathematically defined fractal objects as well as to realworld structures (as, e.g., given in 3-dimensional imaging).

At first we report about experiments carried out on the (up to three levels of recursion) Menger sponge, and also for two fractal variations, generated using slightly modified 1-recursion Menger sponges

Menger sponges of levels of recursion 1, 2, and 3 are shown in the left-most column of Fig. 17. Cell complexes and cycles were automatically computed using the mentioned HSF techniques, and are shown in the other two columns of this figure. The numbers of cycles are given by the formulas in Section 4, and are listed in the third row of Table 1. Visualizations use the program [24].

Further experiments were performed with fractal modifications of the Menger sponge. The initial structures used to generate these new fractals are as follows: 

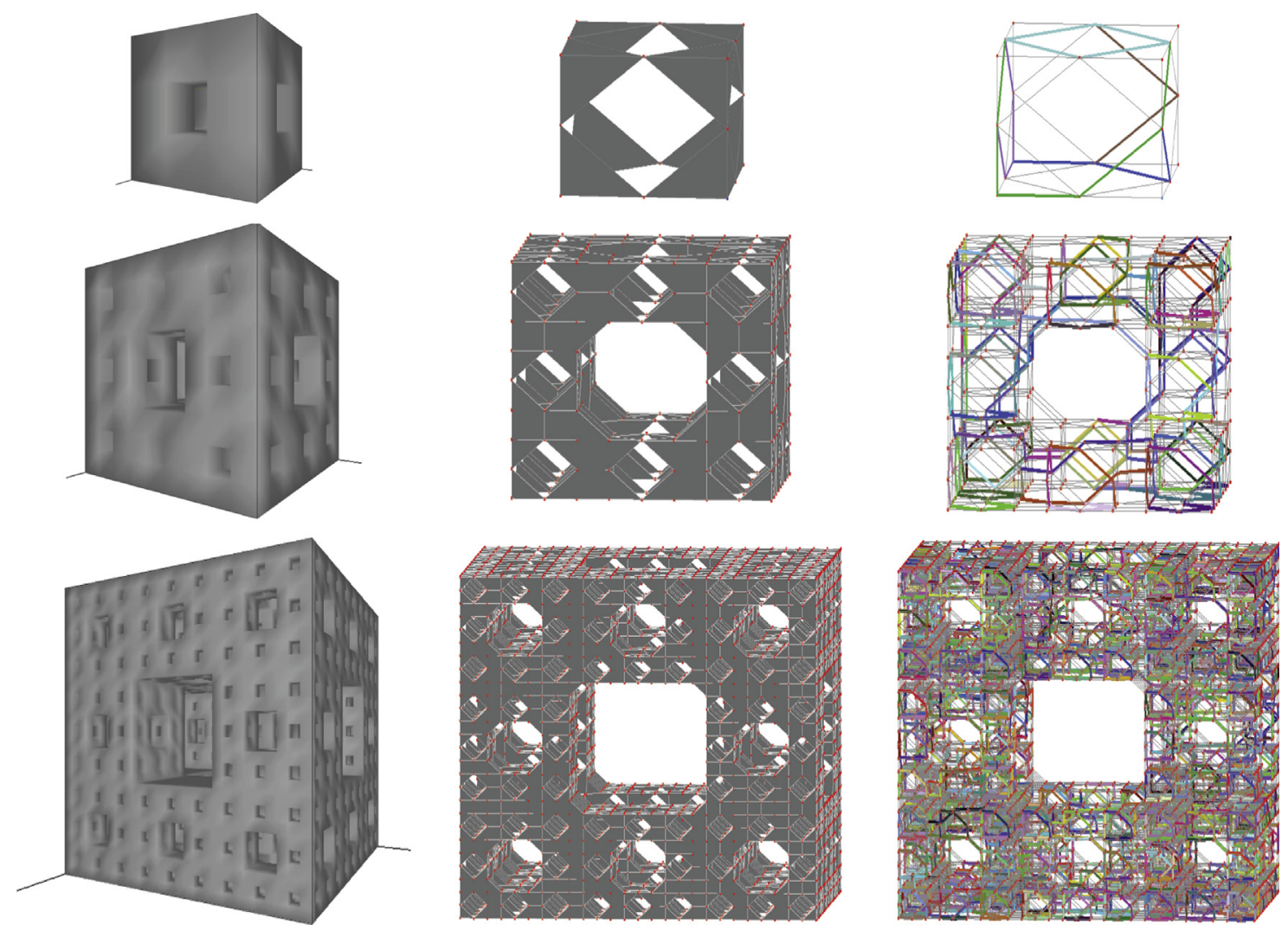

Fig. 17. From left to right: Menger sponge of recursion levels 1, 2 and 3, and their corresponding cell complexes and representative cycles of tunnels.

Table 1

Number of cycles of the Menger sponge and two possible variations for levels of recursion 1,2 , and 3 .

\begin{tabular}{lllr}
\hline Menger variation & Level 1 & Level 2 & Level 3 \\
\hline MengerX1Y3 & 3 & 39 & 590 \\
MengerY3 & 4 & 60 & 1337 \\
Menger & 5 & 81 & 1409 \\
\hline
\end{tabular}

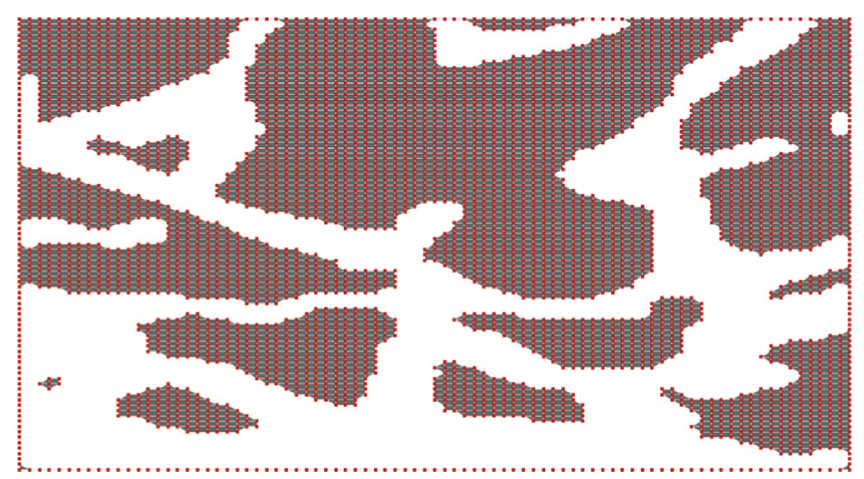

Fig. 18. Cell complex of a trabecular bone slice.

Fractal MengerX1Y3: A variation of the Menger sponge of recursion level 1 in which the OX plane for $x=1$ and the OY plane for $y=3$ are filled.

Fractal MengerY3: A variation of the Menger sponge of recursion level 1 , in which the OY plane for $y=3$ is filled.

Such simple fractal modifications may lead to considerable reductions in the number of cycles. We have computed the cycles for levels 1,2, and 3 of recursion of these modified fractals, and the results are shown in Table 1.

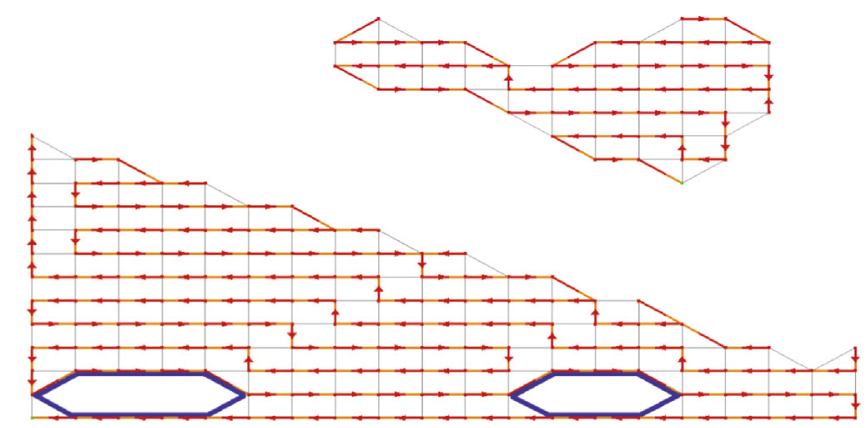

Fig. 19. Zooming into the HSF structure (level 0-1) and representative cycles (in purple) of the trabecular bone example shown in Fig. 18. (For interpretation of the references to color in this figure caption, the reader is referred to the web version of this paper.)

As a second experimental illustration of the proposed techniques, we report about experiments for the analysis of porous materials and fractal objects in real applications in which the data to be analysed has a fractal nature. Examples for such applications are the analysis of trabecular bone images or of human vasculature images; see [2] and [27]. Our initial experiments in this area verify the possibility to compute HSF structures and cycles in one slice of micro-CT trabecular bone images as shown in Figs. 18 and 19. More precisely, these flexible HSF structures topologically describe trabecular bone microarchitectures and are susceptible to be used as a representation for applying simulations to test the fragility of the microarchitecture. A fracture is seen as a topological change; see [21]. Taking into consideration different material properties (bone volume fraction, porosity, connectivity number, fractal dimension, etc.) and searching for statistical dependences between these properties and topological features extracted from HSF structures, is a problem to be studied in the near future. 


\section{Conclusions}

In this paper, an algebraic topological analysis of a fractal, seen as a finite cell complex, is given in terms of a specific chain homotopy equivalence $\mathrm{h} \chi$ between the object and its homology. In the specific cases of Menger sponge and two variants of Sierpiński pyramids, this chain contraction is constructed here starting from combinatorial scaffoldings, called homological spanning forests. By generalizing the presented graph-based encoding scheme for elementary recursive patterns, it is possible to handle the complex topological phenomenology of general multi-dimensional fractals. Future discussion of the geometric, topological, statistical and physical properties (fractal dimension, lacunarity, percolation, porosity, Betti numbers, ....) of these hierarchical structures for fractals will improve the power of the topological analysis applied to a wide range of applications related to fractal dynamical systems, complex networks and physical and biomechanical processes.

\section{Conflict of interest statement}

None declared.

\section{Acknowledgment}

The first and second author have been funded by MICINN under the Feder Funds project 4D-Hom MTM2009-12716.

\section{Appendix A. Supplementary material}

Supplementary data associated with this article can be found in the online version at http://dx.doi.org/10.1016/j.patcog.2014.05.016.

\section{References}

[1] D. Barnes, L. Lambe, Fixed point approach to homological perturbation theory, Proc. Am. Math. Soc. 112 (1991) 881-892.

[2] C.L. Benhamou, E. Lespessailles, G. Acquet, R. Harba, R. Jennane, T. Loussot, D. Tourliere, W. Ohley, Fractal organization of trabecular bone images on calcaneus radiographs, J. Bone Miner. Res. 9 (1994) 1909-1918.

[3] A. Di Carlo, A. Paoluzzi, V. Shapiro, Linear algebraic representation for topological structures, Comput.-Aided Des, 46 (2014) 269-274.

[4] A. Di Carlo, F. Milicchio, A. Paoluzzi, V. Shapiro, Chain-based representations for solid and physical modeling, IEEE Trans. Autom. Sci. Eng. 6 (2009) 454-467.
[5] S. Eilenberg, S. MacLane, On the groups $H(\pi, n)$. Parts I, II, and III, Ann. Math 58, 60, $60(1953,1954) 55-106,48-139,513-557$.

[6] D. Eppstein, Dynamic generators of topologically embedded graphs, in: Proceedings of ACM-SIAM Symposium on Discrete Algorithms, 2003, pp. 599-608.

[7] J. Erickson, K. Whittlesey, Greedy optimal homotopy and homology generators, in: Proceedings of ACM-SIAM Symposium on Discrete Algorithms, 2005, pp. 1038-1046.

[8] R. Forman, Morse theory for cell complexes, Adv. Math. 134 (1998) 90-145.

[9] R. González-Díaz, B. Medrano, P. Real, J. Sanchez-Pelaez, Algebraic topological analysis of time-sequence of digital images, in: Proceedings of International Workshop on Computer Algebra Scientific Computing, Lecture Notes in Computer Sciences, vol. 3718, 2005, pp. 208-219.

[10] R. González-Díaz, P. Real, On the cohomology of 3d digital images, Discret. Appl. Math. 147 (2005) 245-263.

[11] V. Gugenheim, L. Lambe, J. Stasheff, Perturbation theory in differential homological algebra. Parts I and II, Ill. J. Math. 33, 35 (1989, 1991) 566-582 359-373.

[12] T. Kaczynski, K. Mischaikow, M. Mrozek, Computational Homology, Applied Mathematical Sciences, vol. 157, Springer-Verlag, New York, 2004.

[13] R. Klette, A. Rosenfeld, Digital Geometry - Geometric Methods for Digital Picture Analysis, Morgan Kaufmann, San Francisco, 2004.

[14] T. Lewiner, H. Lopes, G. Tavares, Visualizing Forman's discrete vector field, in: Proceedings of International Workshop on Visualization Mathematics, Springer, Heidelberg, 2002, pp. 95-112.

[15] J.B. Listing, Der Census räumlicher Complexe oder Verallgemeinerungen des Euler'schen Satzes von den Polyëdern, Abh. Math. Cl. K. Gesellschaft Wiss. Gött. 10 (1862) 97-182.

[16] K. Menger, Allgemeine Räume und Cartesische Räume, Teil I, Amsterdam, 1926, pp. 476-482.

[17] H. Molina-Abril, P. Real, Homological optimality in discrete Morse theory through chain homotopies, Pattern Recognit. Lett. 33 (2012) 1501-1506.

[18] H. Molina-Abril, P. Real, Homological spanning forest framework for 2D, Ann. Math. Artif. Intell. 64 (2012) 385-409.

[19] M. Mrozek, A. Zelawski, S. Gryglewski, S. Han, A. Krajniak, Homological methods for extraction and analysis of linear features in multidimensional images, Pattern Recognit. 45 (2012) 285-298.

[20] P. Pilarczyk, P. Real, Computation of cubical homology, cohomology, and (co) homological operations via chain contraction, Adv. Comput. Math., 2014, http://dx.doi.org/10.1007/s10444-014-9356-1, in press.

[21] L. Pothuaud, C.L. Benhamou, P. Porion, E. Lespessailles, R. Harba, M.D. Levitz, Fractal dimension of trabecular bone projection texture is related to threedimensional microarchitecture, J. Bone Miner. Res. 15 (2000) 691-699.

[22] V. Robins, J.D. Meiss, E. Bradley, Computing connectedness: an exercise in computational topology, Nonlinearity 11 (1998) 913-922.

[23] V. Robins, J.D. Meiss, E. Bradley, Computing connectedness: disconnectedness and discreteness, Physica D 139 (1998) 276-300.

[24] J.M. Sanchez-Pelaez, 〈munkres.us.es:8080/groups/catam/wiki/e33d2/VOXELO. html, 2007.

[25] F. Sergeraert, The computability problem in algebraic topology, Adv. Math. 104 (1994) 1-29.

[26] W. Sierpiński, Sur une courbe dont tout point est un point de ramification, C.R. Acad. Sci. Paris 160 (1915) 302-305.

[27] G.N. Thomas, S.Y. Ong, Y.C. Tham, W. Hsu, M.L. Lee, Q.P. Lau, W.T. Tay, J. AlessiCalandro, L.A. Hodgson, R. Kawasaki, T.Y. Wond, C.Y. Cheung, Measurement of the macular vascular fractal dimension using a computer-assisted program, Invest. Ophthalmol. Vis. Sci. (2014).

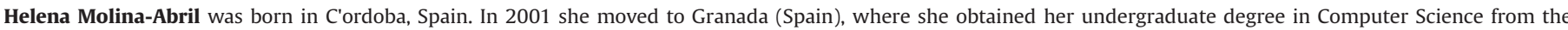

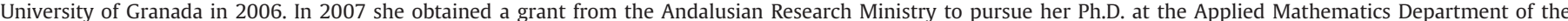

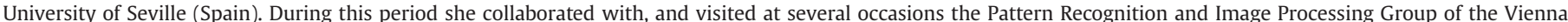

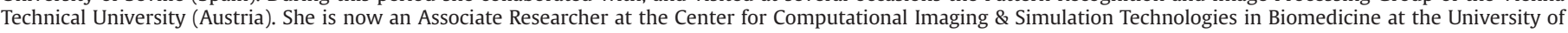
Sheffield (United Kingdom).

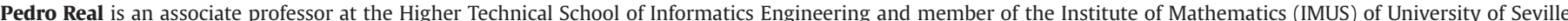

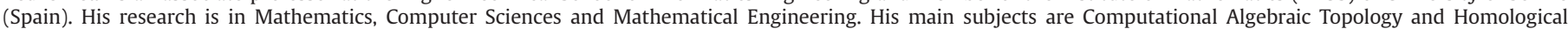

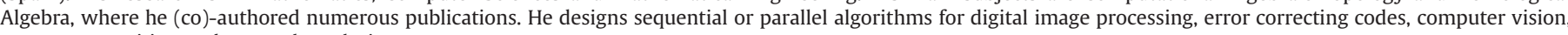
pattern recognition and network analysis.

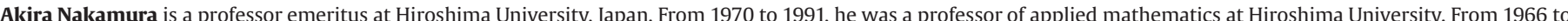

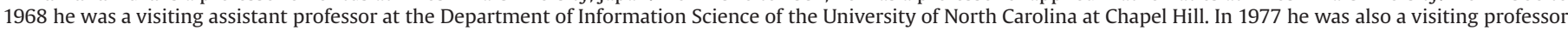

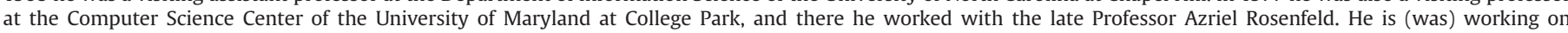

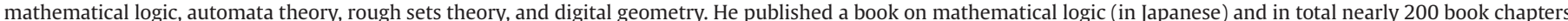

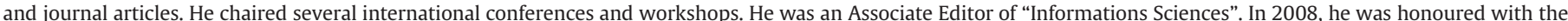
Order of the Sacred Treasure from the Emperor of Japan for his longtime teaching at universities and his outstanding research.

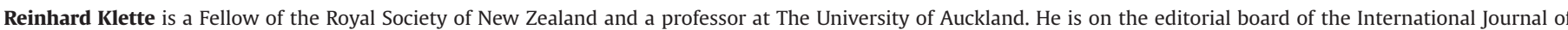

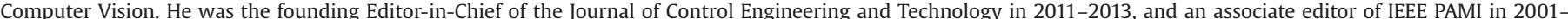

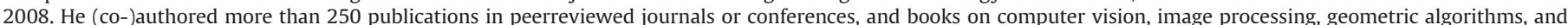

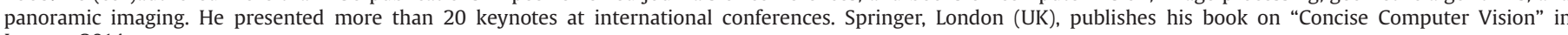
January 2014 